\title{
A Visualization of Air Pollution Distribution over an Observed Area Surrounded by Mountains: A Computational Approach
}

\author{
Udomsak RAKWONGWAN ${ }^{1, *}$, Piyanut TANGMANUSSUKUM ${ }^{2}$ and \\ Sanae RUJIVAN ${ }^{2,3}$
}

\author{
${ }^{I}$ Department of Mathematics, Faculty of Science, Kasetsart University, Bangkok 10900, Thailand \\ ${ }^{2}$ Division of Mathematics and Statistic, School of Science, Walailak University, \\ Nakhon Si Thammarat 80160, Thailand \\ ${ }^{3}$ Center of Excellence in Data Science for Health Study, Walailak University, \\ Nakhon Si Thammarat 80160, Thailand
}

('Corresponding author's e-mail: udomsak.ra@ku.th)

Received: 9 April 2020, Revised: 23 December 2020, Accepted: 30 December 2020

\begin{abstract}
We study the propagation of pollutants emitted from a single generator such as a factory chimney located between 2 mountains as well as its effects on an observed area such as a village or agricultural land. The problem is formulated as a system of partial differential equations, composed of Navier-Stokes equations and an advection-diffusion equation, and is solved by the finite element method. We visualize the propagation of the pollutants for several variants of the problem depending on the heights of the mountains and investigate their negative effects on the observed area by computing an average concentration of the pollutants over the observed area. We found that the observed area between the two mountains experienced a long-term negative effect compared with those located on flat land. This is because the mountain on the side, where the wind is blowing, obstructs the wind resulting in air recirculation. In contrast, the other mountain bounces some pollutants back to the observed area, preventing them from leaving the domain. The higher the mountains, the longer the time the pollutants remain in the observed area. If the heights of the mountains encircling the observed area are not equal, the residual remains in the area longer if the taller mountain is on the windward side.
\end{abstract}

Keywords: Air pollution visualization, Air pollution management, Advection-diffusion equation, NavierStokes equations, Finite element method

\section{Introduction}

Over the past decades, air pollution has increased negatively, affecting individuals and making the world hotter and overpopulated. Not only does it worsen people's health, but it also deals with massive damages to agricultural production. Air pollution is one of the major causes of health issues, both acute and chronic [1]. According to World Health Organization [2], around one-third of deaths from stroke, lung cancer, and heart disease is caused by air pollution, and 9 out of 10 people are breathing polluted air which results in an estimate of 7 million deaths each year. Air pollution's harmful effects on agricultural production have been extensively studied in [3-5]. It stunts the growth of the plants, damages the production, or even kills them off.

Due to an urgent need for air pollution control measures, there have been numerous works on air pollution control. Ghani, Gueraoui, and Men-La-Yakhaf [6] visualized the propagation of air pollutants subject to the wind direction. They modeled the propagation of the air pollutants by Navier-Stokes 
equations [7,8]. The Bouyant force exerting from the temperature change was also considered by integrating the Navier-Stokes equations with Boussinesq approximation. Pochai [9] studied the flow of the air pollutants released by industrial factories through obstacles. He formulated the problem using the stream function and the vorticity equation and solved it by the finite element method, FEM [10-12].

Thus, this paper studies the propagation of the air pollutants emitted by a single generator, such as a factory chimney, surrounded by two mountains in an observed area. Intuitively, the heights of the mountains should have considerable effects on the propagation as they obstruct the wind, which results in the change of its direction. We investigated how the observed area is affected by the pollution by visualizing the propagation of the pollutants over time. We also computed an average of the air pollutants over the area for various cases depending on the heights of the mountains. The observed area can be regarded as a village or agricultural land. The problem was first formulated as a mathematical model and then was numerically solved using FEM. Despite numerous relating works carried out in the last many decades, the investigation of air pollution propagation and its effects on a large-scale area surrounded by tall objects is still lacking.

The organization of this paper is as follows. The "Materials and methods" section describes the mathematical model and all five studied cases depending on the heights of the mountains. It also describes the numerical methods used to solve the problem and the computation of an average concentration over the observed area. The "Results and discussion" section specifies the domain, the initial conditions, and boundary conditions and then shows the visualizations of the air pollution distributions and average concentrations of the pollutants over the observed area for all cases. The last section is "Conclusions".

\section{Materials and methods}

We study the distribution of the pollutants emitted from a single generator $\Omega_{i n}$ which is located between the 2 mountains as seen in Figure 1 and their effects over an observed area $\Omega_{0}$. The domain of the problem $\Omega$ is 2-dimensional and rectangular, and $\Gamma_{1}-\Gamma_{8}$ are the boundaries.

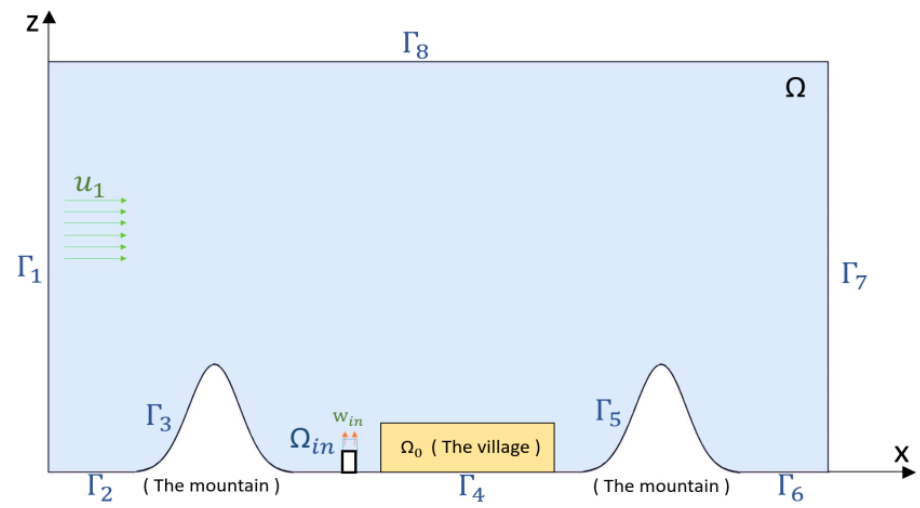

Figure 1 The domain of the problem $\Omega$.

\section{The model}

The propagation of the pollutants can be seen as a fluid dynamics problem, which involves the calculation of various fluid properties such as velocities in $\mathrm{x}$ and $\mathrm{z}$ directions, a pressure, as well as a concentration of the pollutants in the contaminated fluid. The mathematical model used to describe such problem can be expressed as the following system of partial differential equations. 


$$
\begin{aligned}
& \frac{\partial u}{\partial t}+u \frac{\partial u}{\partial x}+w \frac{\partial u}{\partial z}=-\frac{1}{\rho} \frac{\partial p}{\partial x}+\frac{\mu}{\rho}\left(\frac{\partial^{2} u}{\partial x^{2}}+\frac{\partial^{2} u}{\partial z^{2}}\right) \\
& \frac{\partial w}{\partial t}+u \frac{\partial w}{\partial x}+w \frac{\partial w}{\partial z}=-\frac{1}{\rho} \frac{\partial p}{\partial x}+\frac{\mu}{\rho}\left(\frac{\partial^{2} w}{\partial x^{2}}+\frac{\partial^{2} w}{\partial z^{2}}\right)+g \\
& \frac{\partial u}{\partial x}+\frac{\partial u}{\partial z}=0 \\
& \frac{\partial C}{\partial t}+u \frac{\partial C}{\partial x}+w \frac{\partial C}{\partial z}=\frac{\partial}{\partial x} K_{x} \frac{\partial C}{\partial x}+\frac{\partial}{\partial z} K_{z} \frac{\partial C}{\partial z}
\end{aligned}
$$

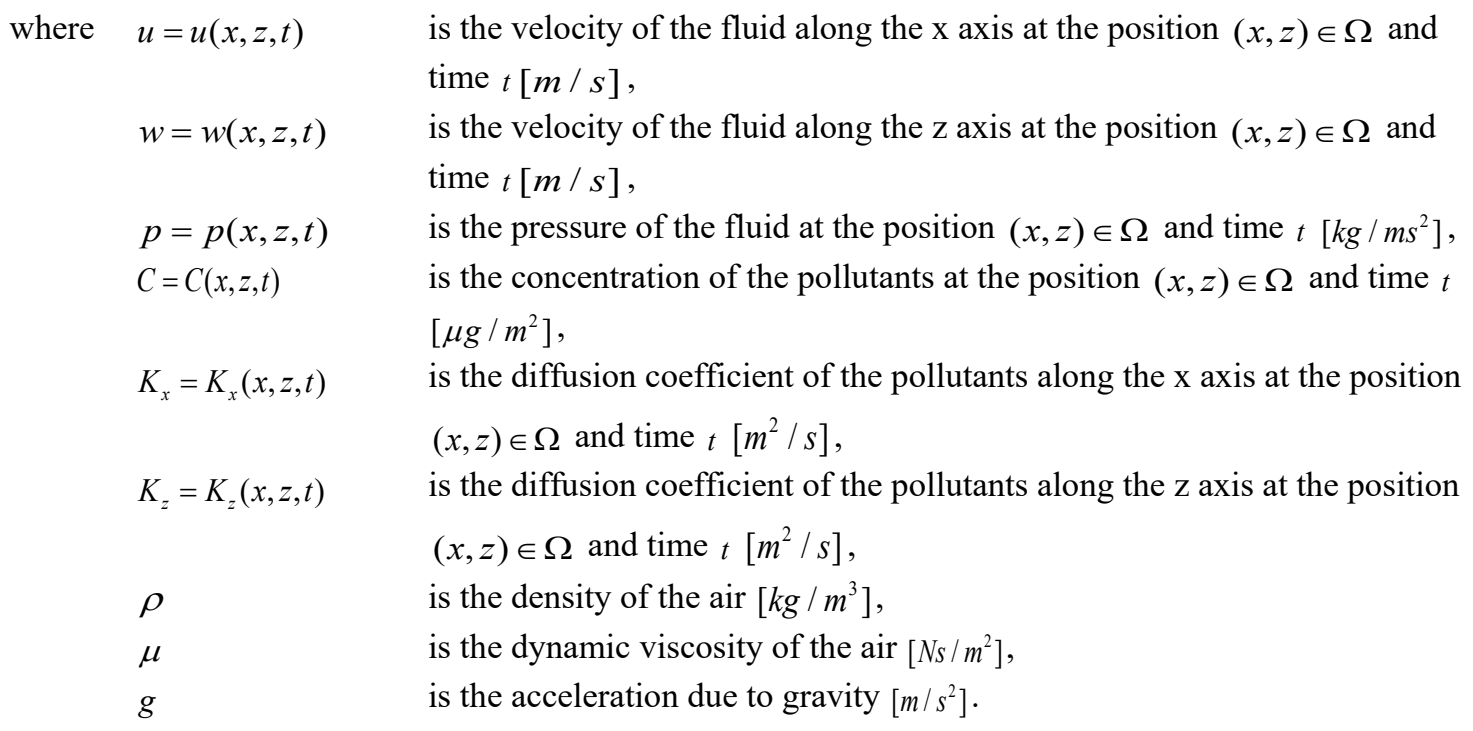

The flow of the air is described by Navier-Stokes Eqs. (1) - (3) derived from the laws of conservation of mass, momentum, and energy [7,8]. The propagation of the pollutants is described by Eq. (4) which is an advection-diffusion equation [13]. The air is assumed incompressible.

\section{Initial conditions and boundary conditions}

Initial conditions

The pollutants are assumed to be emitted only once at the beginning of the computation with the concentration $C_{i n}$ which is a function of a position along the generator's boundary. In other words, the pollutant generator does not continuously produce the pollutants over time. The cases for continuously or periodically emitting can be done easily by modifying the generator's boundary condition. We also assume that there is wind blowing out vertically along with the pollutants at the speed $w_{i n}$. Mathematically, for a position $(x, z)$ along the generator's boundary $\Omega_{i n}$, or $(x, z) \in \Omega_{i n}$,

$u(x, z, 0)=0$,

$w(x, z, 0)=w_{i n}(x, z)$, 
$C(x, z, 0)=C_{i n}(x, z)$,

where $w_{i n}(x, z) \quad$ is the velocity of the fluid along the $\mathrm{x}$ axis a position $(x, z)$ at $t=0$,

$C_{i n}(x, z) \quad$ is the concentration of the pollutants at a position $(x, z)$ at $t=0$.

For other positions in the domain, $(x, z) \in \Omega$, we have that

$u(x, z, 0)=u_{0}(x, z)$

$w(x, z, 0)=w_{0}(x, z)$,

$C(x, z, 0)=C_{0}(x, z)$,

where $u_{0}(x, z) \quad$ is the velocity of the fluid along the $\mathrm{x}$ axis at a position $(x, z)$ at $t=0$,

$w_{0}(x, z) \quad$ is the velocity of the fluid along the $y$ axis at a position $(x, z)$ at $t=0$,

$C_{0}(x, z) \quad$ is the concentration of the pollutants at the position $(x, z)$ at $t=0$.

\section{Boundary conditions}

There are 8 boundaries in the problem. The wind is flowing into the domain from the boundary $\Gamma_{1}$ in the $\mathrm{x}$ direction with a speed of $u_{1}$ along with pollutants with a concentration of $C_{1}$. The boundaries $\Gamma_{3}$ and $\Gamma_{4}$ are those of the mountains which assume to bounce back the wind and the pollutants. The boundary conditions can intuitively be express as,

For: $(x, z) \in \Gamma_{1}$

$$
u(x, z, t)=u_{1}(x, z, t), \quad w(x, z, t)=0, \quad C(x, z, t)=C_{1}(x, z, t) .
$$

For $(x, z) \in \cup_{i=2,3,4,5,6,8} \Gamma_{i}:$

$$
u(x, z, t)=0, \quad w(x, z, t)=0, \quad \frac{C(x, z, t)}{\partial z}=0 .
$$

For $(x, z) \in \Gamma_{7}$ :

$$
\frac{u(x, z, t)}{\partial x}=0, \quad \frac{w(x, z, t)}{\partial x}=0, \quad \frac{C(x, z, t)}{\partial z}=0 .
$$

\section{Studied cases}

In order to see the effects of the heights of the mountains on the propagation of the pollutants, we study 5 variants of the problem which are $(0-0),(600-600),(900-900),(300-900)$, and $(900-300)$, where $\left(h_{1}-h_{2}\right)$ denotes the case where the height of the $1^{\text {st }}$ mountain on the left-hand side is $h_{1}$ and the height of the $2^{\text {nd }}$ mountain on the right-hand side is $h_{2}$.

\section{The numerical method}

The solution of the system of partial differential equations is difficult to be obtained analytically. It is more practical to solve the model numerically, especially when 1 varies the shape of the domain, or the initial and boundary conditions to study the effects of those varied factors on the solution. 
There are various computational methods typically used for solving the system of partial differential equations, such as the finite difference method, the finite element method [10-12], the finite volume method and the lattice Bolzman method [14]. Each method has its own pros and cons. In this paper, we use the software called "the distributed and unified numeric environment" or DUNE $[15,16]$ which employs FEM to solve systems of partial differential equations. The domain $\Omega$ is discretized as in Figure 2. The discretized domain is generated with $\mathrm{Gmsh}$, which is a finite-element mesh generator developed by [17]. It can be seen that the domain is not equally discretized which is one of the advantages of FEM. This is very helpful as one can only discretize the focused area into small pieces. The area which is not important can be discretized into bigger pieces. This results in great reduction in the computational time.

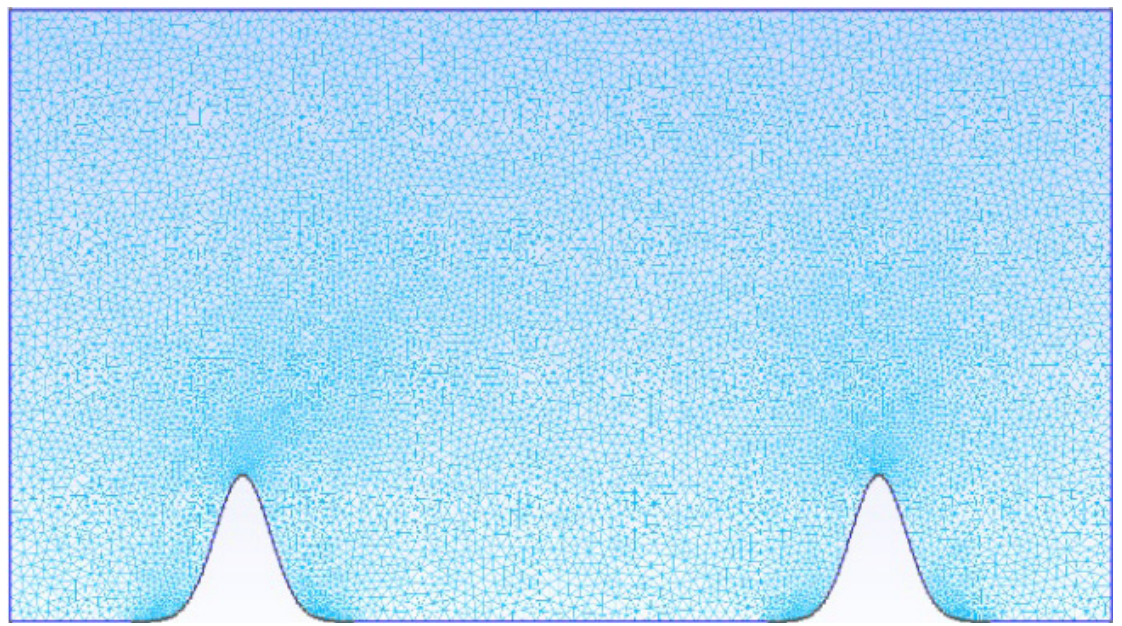

Figure 2 Discretized domain for the finite element method.

The average concentration of the pollutants in the observed area

The average concentration of the pollutants over the observed area can be seen as a measure of the negative effects of the air pollution. However, as the domain is not discretized equally for FEM, the computation of the average concentration is not trivial. In this paper, it is approximated by $1^{\text {st }}$ equally discretizing the observed domain into $(m-1) \times(n-1)$ rectangles and computing the mean of the average concentrations of the pollutants in the small rectangles Figure 3. Mathematically, the average of the pollutants over the observed area, $\bar{C}(t)$, as a function of time can be approximated by;

$\bar{C}(t)=\frac{\iint_{(x, z) \in \Omega_{0}} C(x, z, t) d x d z}{\left\|\Omega_{0}\right\|} \approx \frac{\sum_{i=1}^{m-1} \sum_{j=1}^{n-1} C_{i j} \Delta x \Delta z}{\left\|\Omega_{0}\right\|}$

where $\left\|\Omega_{0}\right\| \quad$ is the area of $\Omega_{0}$ which is equal to $l \times h$,

$\Delta x, \Delta z \quad$ are the lengths of the rectangles which equal $l / m$ and $h / n$ respectively,

$C_{i j}(t) \quad$ is an average of the concentration $C(x, z, t)$ over the rectangle $(i, j)$ at time $t$. 


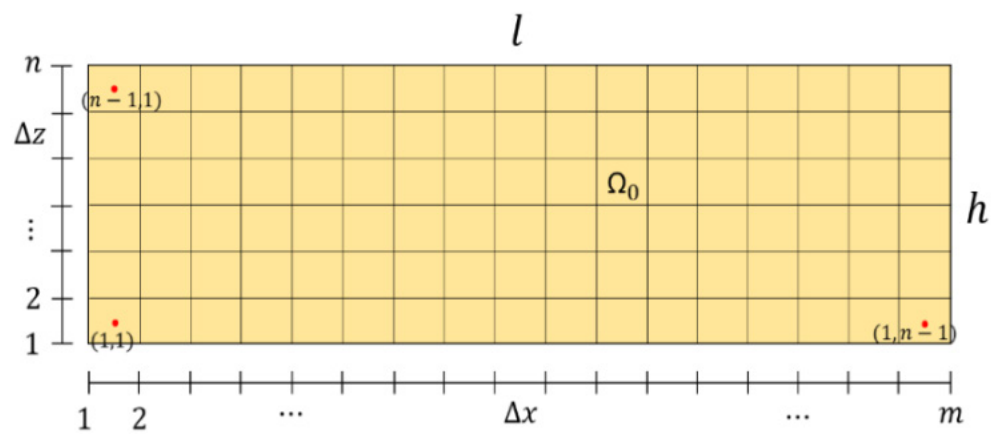

Figure 3 Observed domain $\Omega_{0}$ discretized into $(m-1) \times(n-1)$ rectangles.

\section{Results and discussion}

In this section, we illustrate the technique by visualizing the propagation of the pollutants emitted from a single generator over time as well as computing the average concentration of the pollutants over the observed area which is $200 \mathrm{~m}$ tall and $800 \mathrm{~m}$ wide located between the 2 mountains and $200 \mathrm{~m}$ away from the generator Figure 4. The domain is $4500 \mathrm{~m}$ tall and $2500 \mathrm{~m}$ wide, and the mountains are $900 \mathrm{~m}$ wide. We also consider the 5 variants of the problem mentioned earlier to study how the propagation of the pollutants is affected by the heights of the mountains over time.

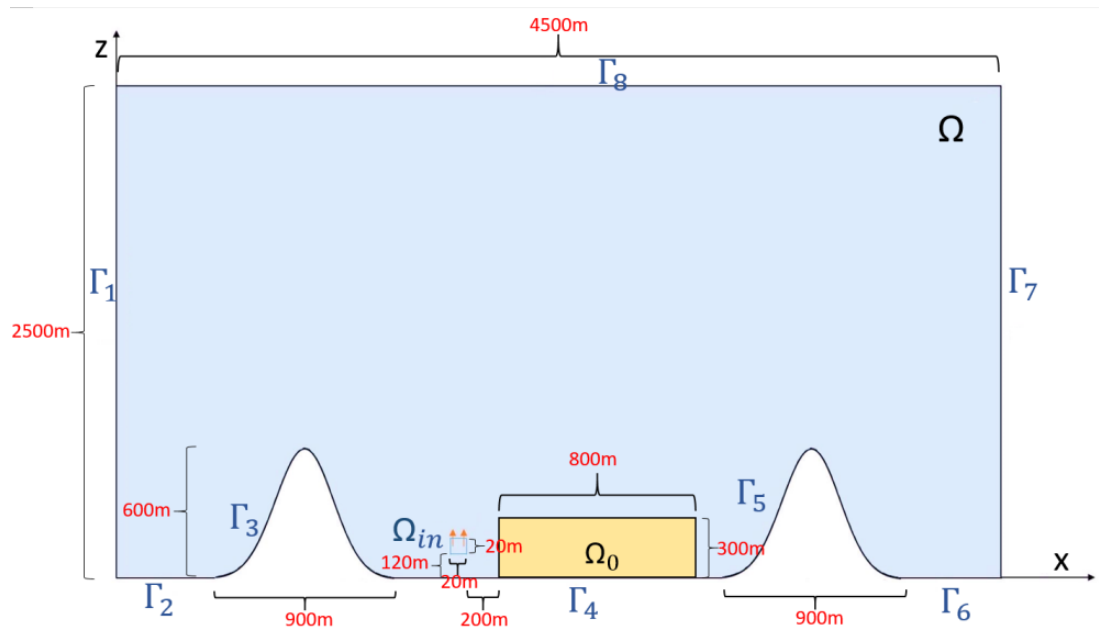

Figure 4 Domain $\Omega$ and the observed area $\Omega_{0}$ with the specified heights and widths.

\section{Parameters, initial and boundary conditions specification}

In order to solve the model, the parameters, the initial, and the boundary conditions need to be specified first. The standard values of the density $\rho$ and the dynamic viscosity $\mu$ of the air at $300 \mathrm{~K}$ are $1.184 \mathrm{~kg} / \mathrm{m}^{3}$ and $1.849 \mathrm{Ns} / \mathrm{m}^{2}$ respectively [18]. The value of the diffusion coefficient along the $\mathrm{x}$ axis, $K_{x}$, is assumed to be 0 as we assume that the effect of the diffusion on the propagation in the horizontal direction is negligible compared to that of the wind. In other words, $u \frac{\partial C}{\partial x}>>\frac{\partial}{\partial x} K_{x} \frac{\partial C}{\partial x}$. The vertical diffusion coefficient, however, from [19], can be written as; 
$K_{z}=K_{z}(z)=0.4 u_{*} z e^{-\frac{z}{H}}$,

where a fractional velocity $u_{*}$ and an average height $H$ of the surface at the bottom of the domain are $0.16 \mathrm{~m} / \mathrm{s}$ and $1 \times 10^{-5} \mathrm{~m}$, respectively.

The pollutants are initially emitted from the boundary $\Omega_{i n}$ with the concentration of $1 \mathrm{~g} / \mathrm{m}^{2}$ along with the wind vertically blowing out along with pollutants at the speed of $14 \mathrm{~m} / \mathrm{s}$. In other words, $C_{i n}(x, z)=1 \mathrm{~g} / \mathrm{m}^{2}$ and $w_{i n}(x, z)=14 \mathrm{~m} / \mathrm{s}$ for all $(x, z) \in \Omega_{i n}$. For other positions $(x, z)$ in the domain $\Omega$, $C_{0}(x, z)=0, w(x, z)=0$ and $u(x, z)=u_{0}\left(z / z_{0}\right)^{0.15}$ where $u_{0}$ is the velocity in the horizontal direction at the height of $z_{0}$ [20]. The horizontal velocity at the height of $100 \mathrm{~m}$ is $4.4 \mathrm{~m} / \mathrm{s}$. Thus, $u_{0}=4.4 \mathrm{~m} / \mathrm{s}$ and $z_{0}=$ $100 \mathrm{~m}$.

On the boundary $\Gamma_{1}$, we assume that the wind is flowing into the domain at the speed of $u_{0}\left(z / z_{0}\right)^{0.15}$. We also assume that there are not pollutants flowing into the domain along with the wind or $C_{1}(x, z)=0$.

\section{Results}

This subsection shows the results, which are the visualization of the pollutants as well as its effects on the observed area for the cases mentioned earlier. Figure 5 illustrates the velocity field at 1, 10, 20, 70 and $200 \mathrm{~s}$. The initial velocity in the vertical direction is 0 , whereas that in the horizontal direction is $u(x, z)=u_{0}\left(z / z_{0}\right)^{0.15}$. This is why we see the wind is moving nearly horizontally and has higher speed at the upper region of the domain after $1 \mathrm{~s}$. As time passes, we can see the recirculation zones due to the mountains and the air pressure.

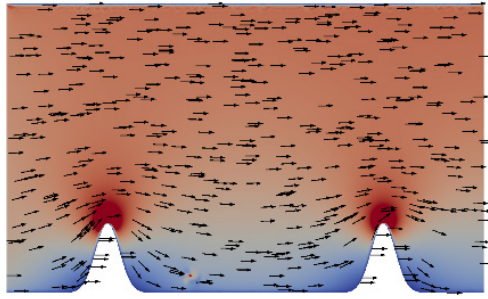

(a)

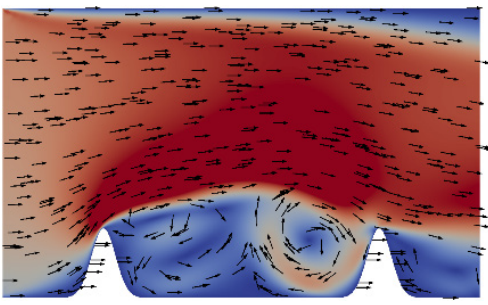

(d)

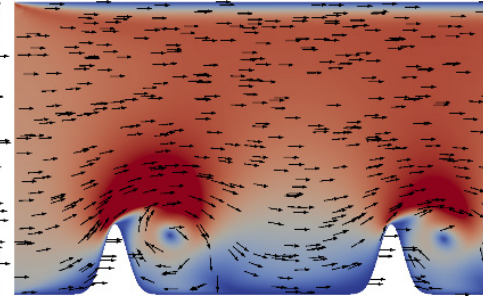

(b)

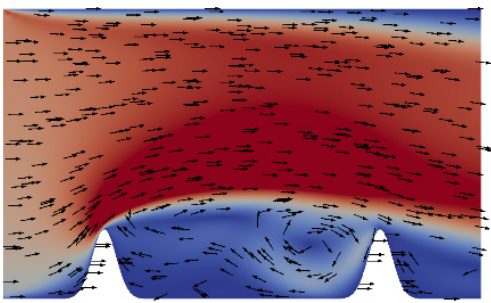

(e)

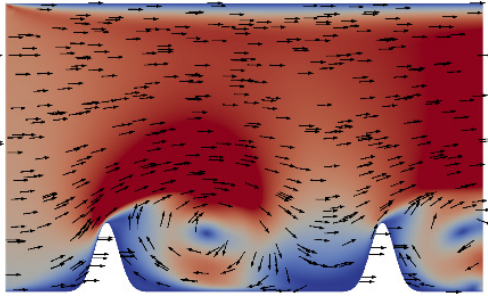

(c)

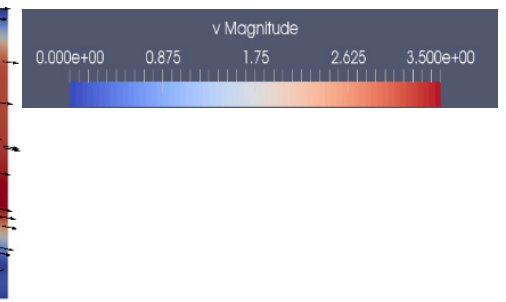

Figure 5 Velocity field at 1 (a), 10 (b), 20 (c), 70 (d), 200 (e) s.

Figure 6 illustrates the concentration of the pollutants for $(0,0)$, where the domain is flat, at 1,10 , 20, 70, and $200 \mathrm{~s}$. The pollutants are initially emitted from the generator and then move to the right side of the domain. They slightly move up at the beginning due to the wind blowing out vertically along with 
the pollutants. We see that in the first $10 \mathrm{~s}$ the pollutants are stick together and do not flow to the righthand side as much as from 10 to $20 \mathrm{~s}$. This is because the wind speed flowing to the right-hand side of the domain is weaker than that in the lower part of the domain. The pollutants are all blown out of the domain by $200 \mathrm{~s}$.

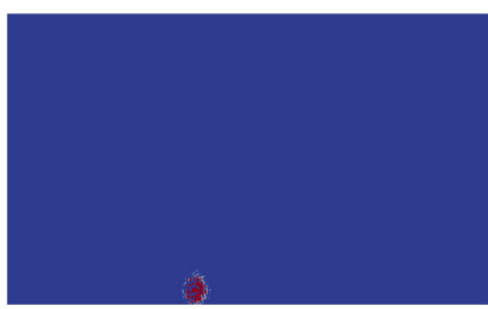

(a)

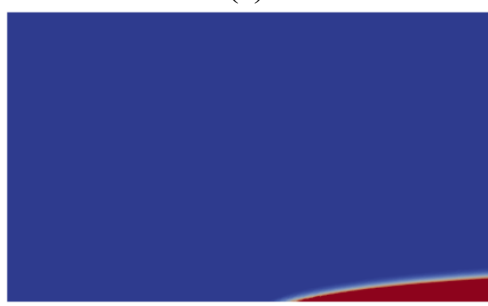

(d)

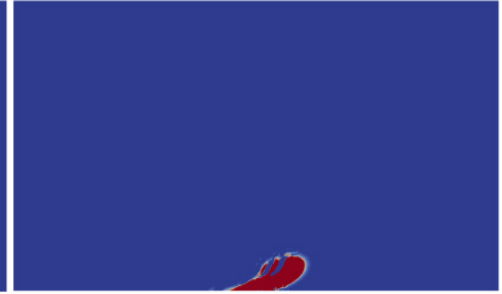

(b)

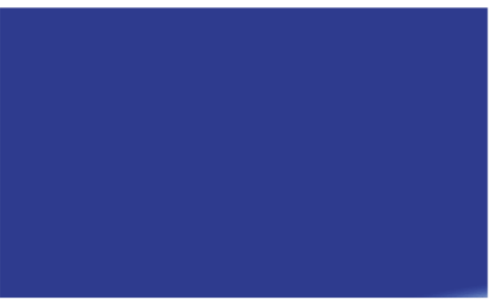

(e)

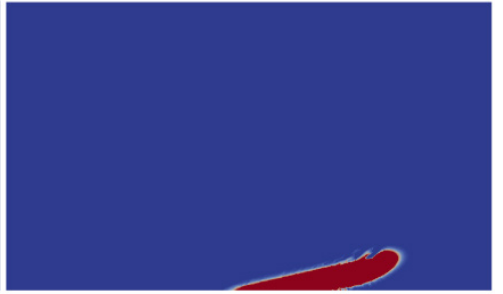

(c)

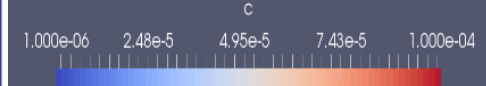

Figure 6 Concentration of the pollutants for $(0,0)$ at 1 (a), 10 (b), 20 (c), 70 (d), 200 (e) s.

The average concentration of the pollutants over the observed domain is shown in Figure 7. We see that the pollutants have reached the observed area at around $4 \mathrm{~s}$. The average concentration jumps up drastically and reaches its peak at around $6 \mathrm{~s}$ and then steeply decreases until $20 \mathrm{~s}$ when it begins to drop gradually. The observed area has stopped experiencing the pollution at around $80 \mathrm{~s}$.

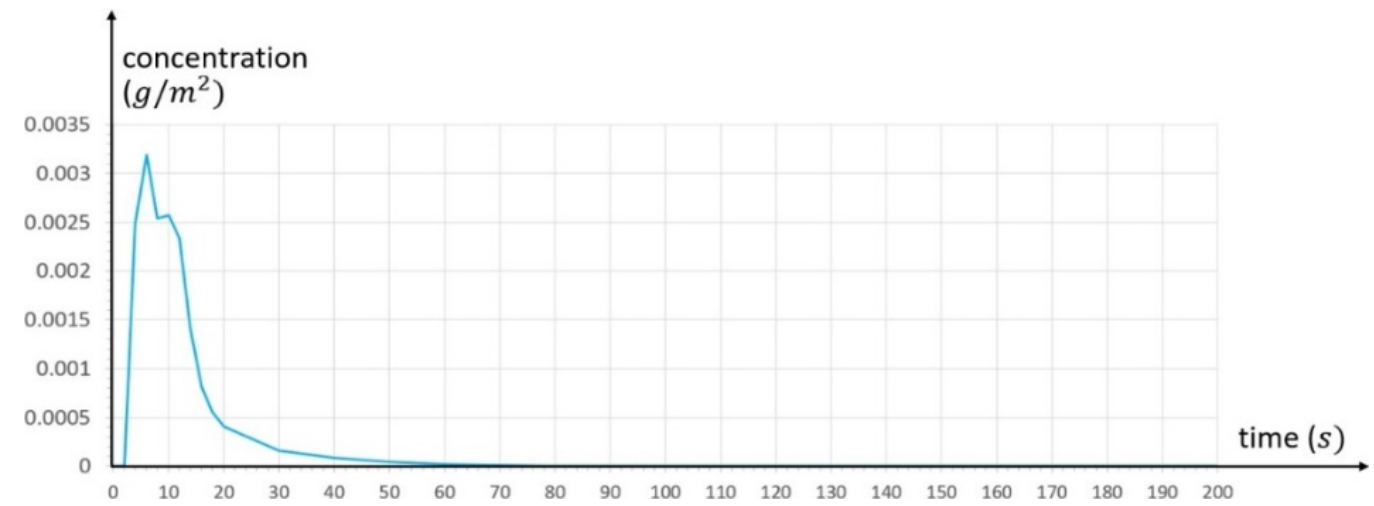

Figure 7 Average concentration of the pollutants over the observed area for $(0,0)$.

Figure 8 shows the concentration of the pollutants for $(300,300)$, where both mountains are $300 \mathrm{~m}$ tall, at $1,10,20,70$, and $200 \mathrm{~s}$. We see that although most pollutants are blown out of the domain over the right-hand side mountain, some pollutants bounced back into the domain. As a result, there are still some pollutants remaining after $200 \mathrm{~s}$. In other words, being surrounded by the mountains at the same height of $300 \mathrm{~m}$ results in having pollutant residue for longer time. 


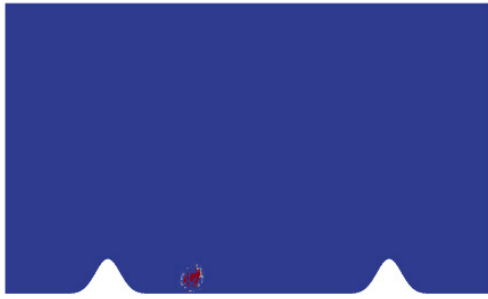

(a)

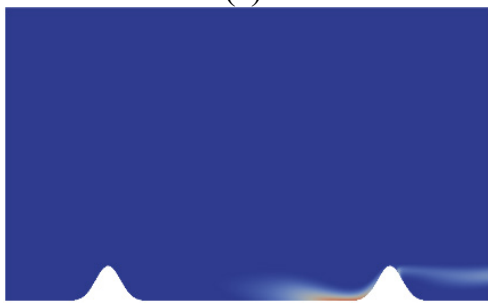

(d)

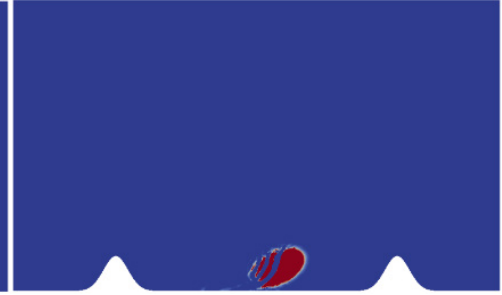

(b)

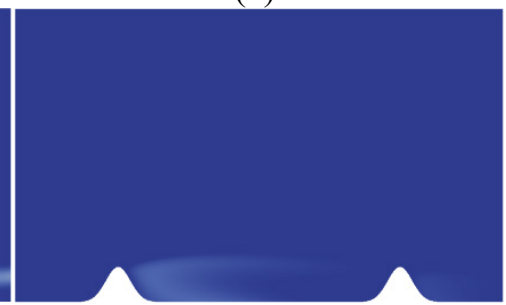

(e)

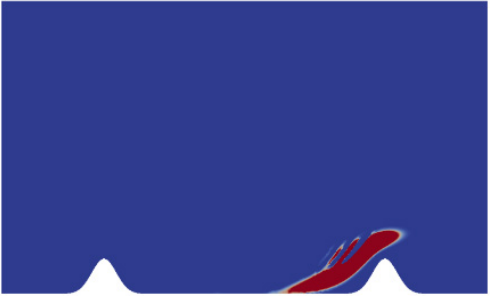

(c)

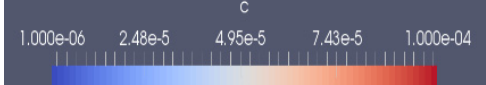

Figure 8 The concentration of the pollutants for $(300,300)$ at 1 (a), 10 (b), 20 (c), 70 (d), 200 (e) s.

Figure 9 shows the average concentration of the pollutants over the observed area as a function of time. We see that, compared with $(0,0)$, a village located between the 2 mountains at the same height of $300 \mathrm{~m}$ experiences the higher peak of the pollutant concentration but has lower average concentration after around $15 \mathrm{~s}$.

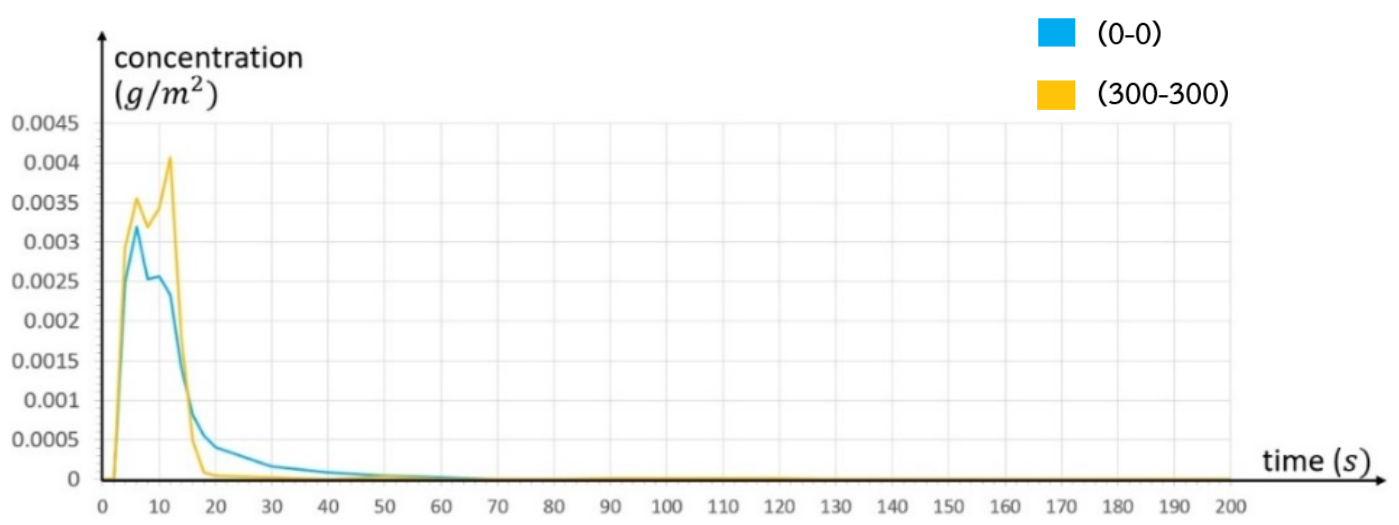

Figure 9 Average concentration of the pollutants over the observed area for $(300,300)$.

To investigate the effects of the heights of the mountains on the propagation of the air pollutants further, we consider a variant of the problem where the height of both mountains is $900 \mathrm{~m}$. Figure 10 shows the concentration of the pollutants for $(900,900)$ at $1,10,20,70$, and $200 \mathrm{~s}$. The propagation is similar to that of $(300,300)$ in the first $10 \mathrm{~s}$. However, after $10 \mathrm{~s}$, the pollutants flow to the left-side of the domain due to the wind recirculation; see Figure 5. The pollutants hit the left mountain first and are bounced back to the right-hand side. As a result, it leaves much more pollutant residue in the domain compared with $(0,0)$, and $(300,300)$ in a long run. 


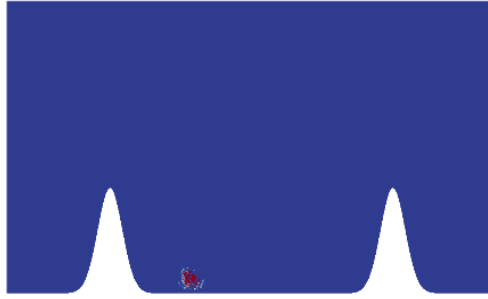

(a)

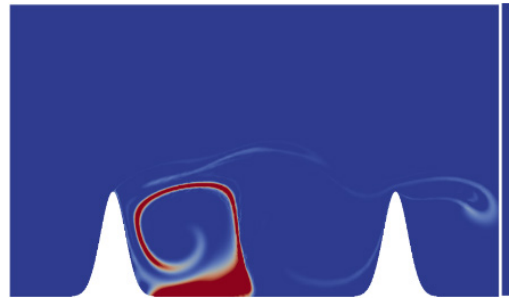

(d)

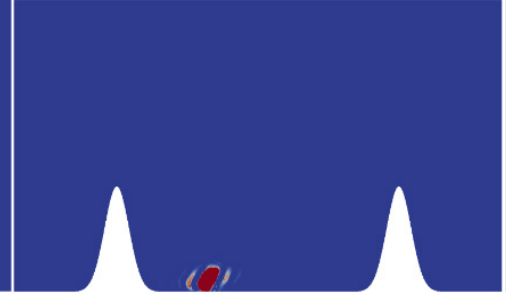

(b)

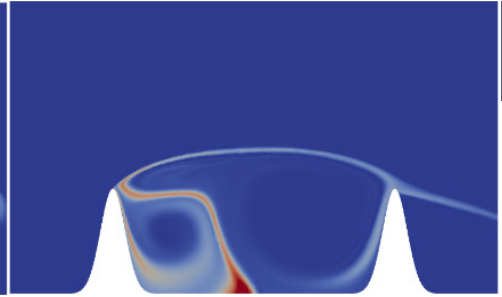

(e)

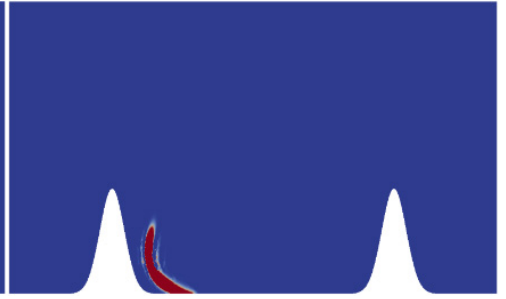

(c)

Figure 10 Concentration of the pollutants for $(900,900)$ at 1 (a), 10 (b), 20 (c), 70 (d), 200 (e) s.

Figure 11 shows the average concentration of the pollutants over the observed area as a function of time. The observed area is not greatly affected by the air pollution until around $30 \mathrm{~s}$. After $30 \mathrm{~s}$, the averaged concentration of the air pollutants over the observed area goes up until $50 \mathrm{~s}$ and starts decreasing very slowly. It would take longer time for the pollutants to dissipate compared with $(0,0)$, and $(300,300)$.

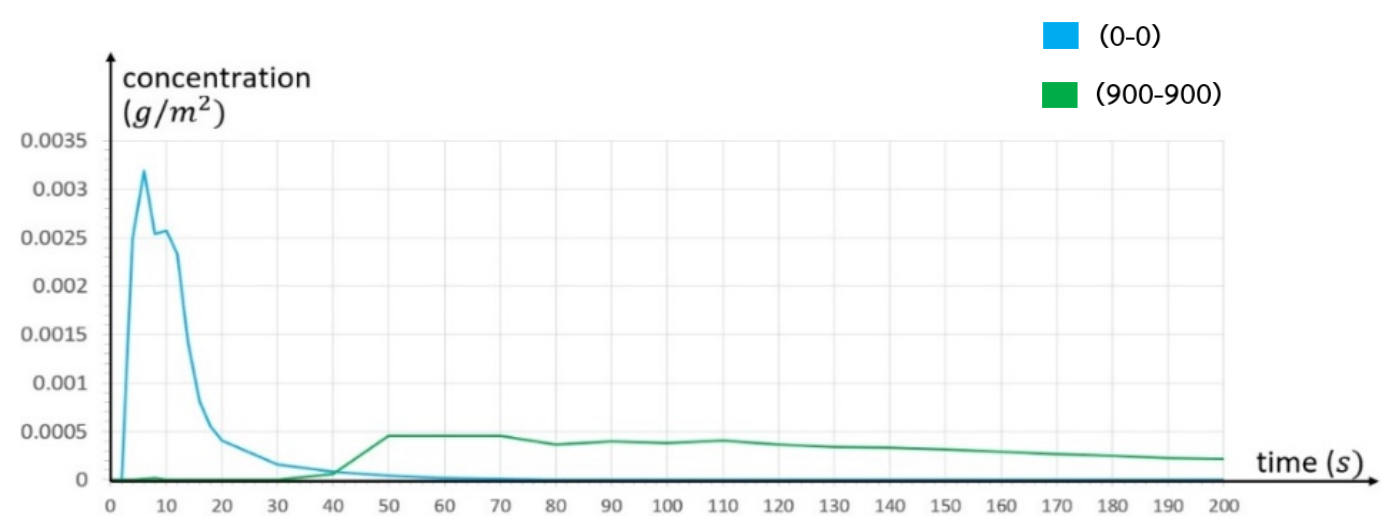

Figure 11 Average concentration of the pollutants over the observed area for $(900,900)$.

Figure 12 shows the propagation of the pollutants for $(300,900)$, where the left mountain is $300 \mathrm{~m}$ tall and the right mountain is $900 \mathrm{~m}$ tall, at 1, 10, 20,70, and $200 \mathrm{~s}$. The propagation in the first $10 \mathrm{~s}$ is similar to that of $(300,300)$ as their heights of the mountain on the left-hand side which is the direction the wind is blowing from are equal. The pollutants initially propagate due to the wind and the diffusion. After hitting the higher mountain on the right-hand side, some of the pollutants bounced back to the middle of the domain. 


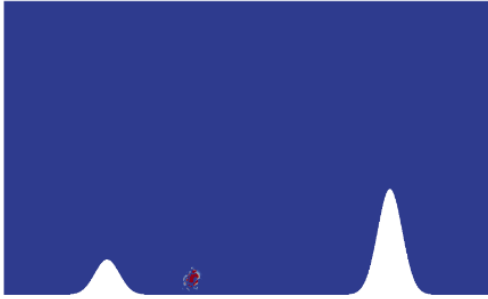

(a)

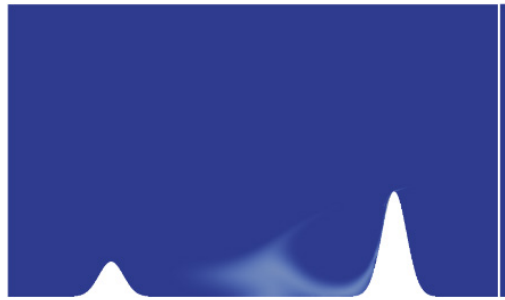

(d)

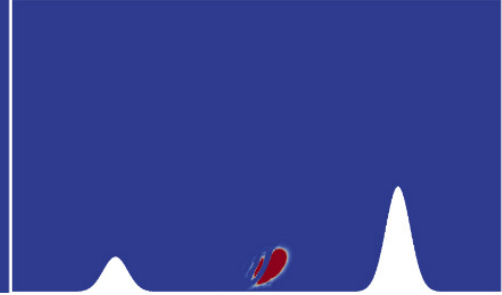

(b)

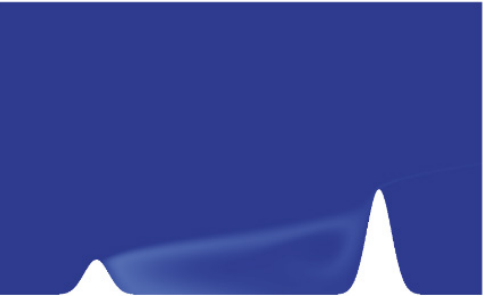

(e)

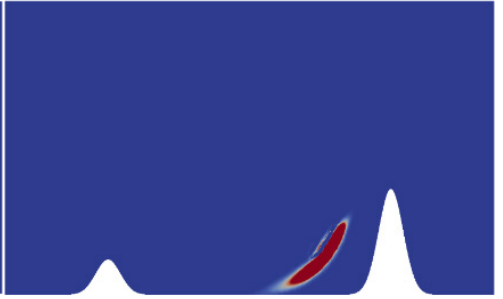

(c)

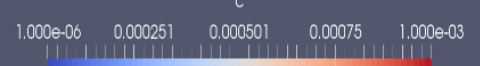

Figure 12 Concentration of the pollutants for $(300,900)$ at 1 (a), 10 (b), 20 (c), 70 (d), 200 (e) s.

Figure 13 shows the average concentration of the pollutants over the observed area as a function of time. We see that the average concentration steeply climbs up to its peak by $8 \mathrm{~s}$. After $8 \mathrm{~s}$, the concentration decreases until near 0 at $30 \mathrm{~s}$. This is when part of the pollutants bounced back by the 900 $\mathrm{m}$ high mountain. As the bounced-back pollutants arrive back in the observed area after around $50 \mathrm{~s}$, the concentration increases again.

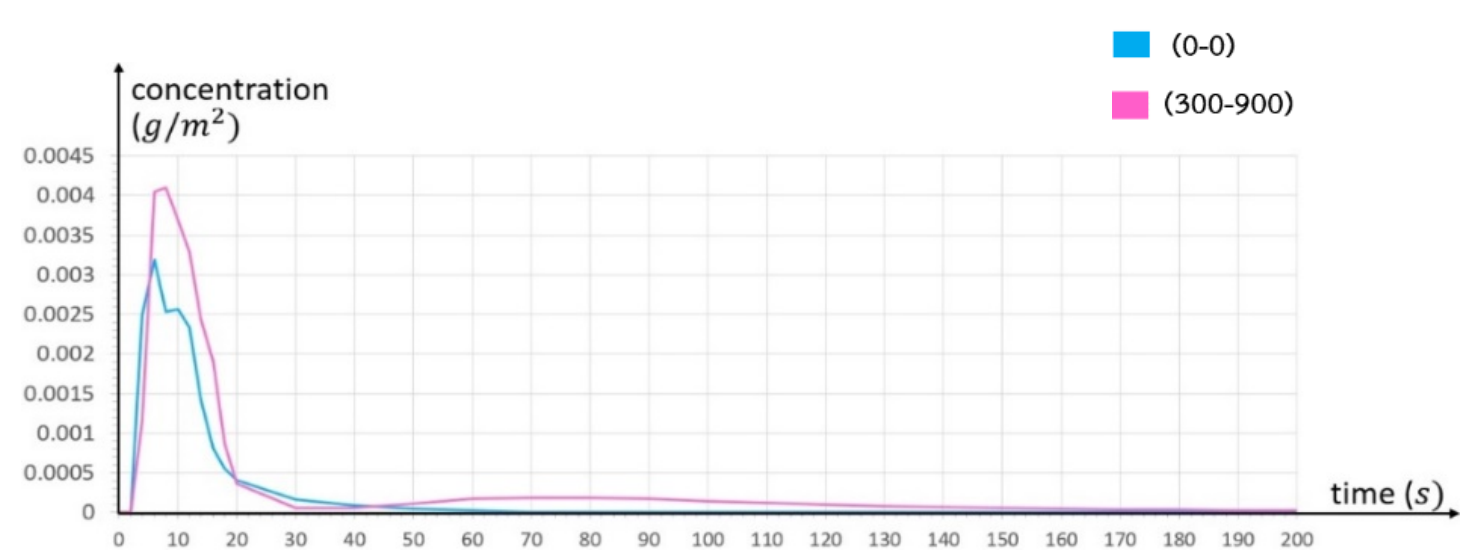

Figure 13 Average concentration of the pollutants over the observed area for $(300,900)$.

Figure 14 illustrates the propagation of the pollutants for $(900,300)$, where the left mountain is $900 \mathrm{~m}$ tall and the right mountain is $300 \mathrm{~m}$ tall, at $1,10,20,70$, and $200 \mathrm{~s}$. We can see that up to $20 \mathrm{~s}$, the propagation of the pollutants is similar to that of $(900,900)$. The reason is similar to the previous case where we compare $(300,900)$ with $(300,300)$. The heights of the left-hand side mountains for both cases are equal. However, after $20 \mathrm{~s}$, $(900,300)$ has less pollutants remaining in the domain compared with $(900,900)$ as more pollutants are blown over the right mountain and leave the domain. 


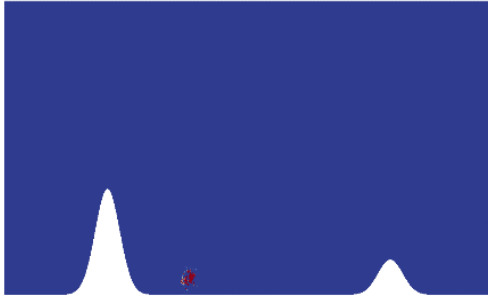

(a)

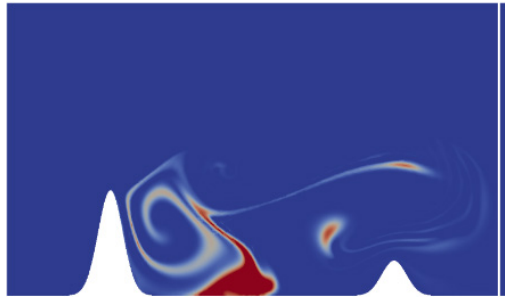

(d)

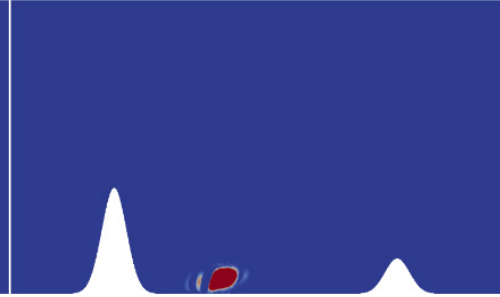

(b)
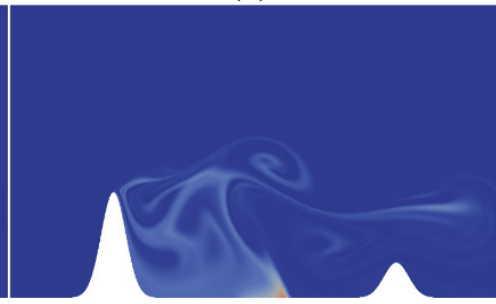

(e)

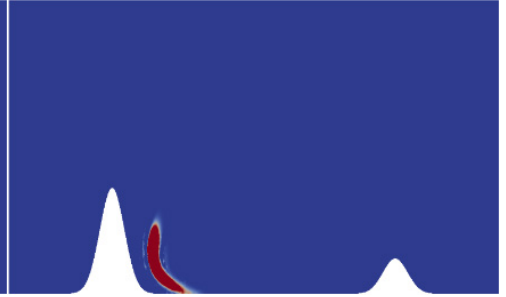

(c)

Figure 14 Concentration of the pollutants for $(900,300)$ at 1 (a), 10 (b), 20 (c), 70 (d), 200 (e) s.

Figure 15 shows the average concentration of the pollutants over the observed area as a function of time. It has similar pattern to that of $(900,900)$ except that the peak concentration of the pollutants at around $8 \mathrm{~s}$ is higher, but compared with $(0,0),(300,300),(600,600)$ and $(300,900),(900,300)$ has higher concentration of the pollutants in a long run.

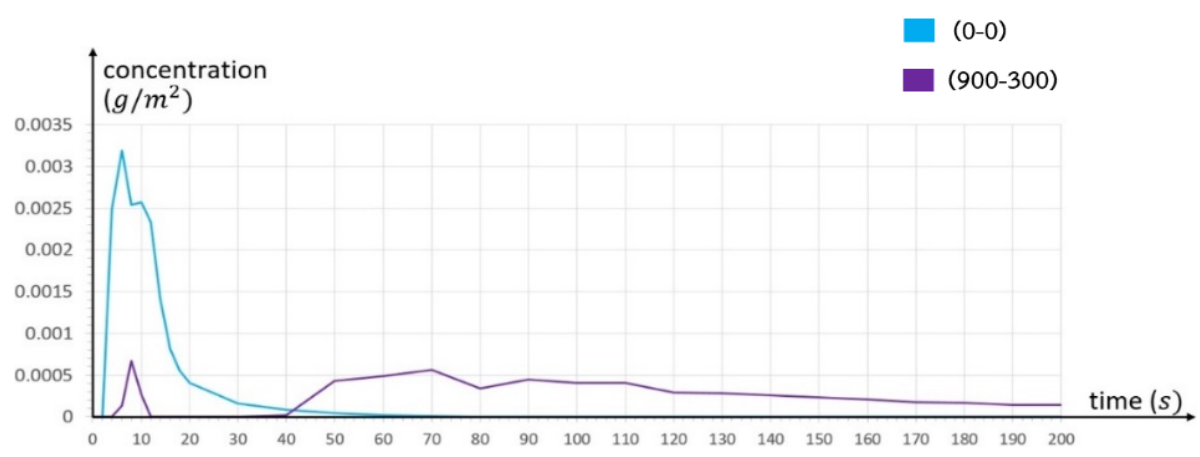

Figure 15 Average concentration of the pollutants over the observed area for $(900,300)$.

\section{Discussion}

We see that the observed areas surrounded by mountains suffer from pollution for a more extended time than those located in flat land. The mountain on the left-hand side, which is where the wind is blowing from, obstructs the wind resulting in air recirculation. The other mountain blocks the wind and prevents some pollutants from leaving the domain.

Having more pollutant residue in the observed area for a long time such as in $(900,900)$ and $(900,300)$ is not always bad. It depends how well the observed area can tolerate the pollution. For example, if the pollution level which starts to negatively affect individuals is $0.001 \mathrm{~g} / \mathrm{m}^{2}$, people living in $(900,900)$ and $(900,300)$ will not be affected by the pollution at all. However, if the time being exposed to the pollution is more critical to the health, they will greatly suffer than those living in the domains in the other cases. 
Many studies on air pollution modeling have employed FEM to solve the problems with a single generator [21-24] or multiple generators [9,25]. [26] visualized the distribution of the pollutants in a street area through obstacles, whereas the simulation on the regional scale was carried out using online-coupled Weather Research and Forecasting with Chemistry (WRF-Chem) model developed collaboratively by several agencies which simultaneously simulates the emission, mixing, transport, trace gases and aerosols [27]. As there have not been many works that studied the air pollution propagation on a wide-ranging land encircled by tall objects, this paper fills this gap.

\section{Conclusions}

As we studied the propagation of the pollutants emitted from a generator located between 2 mountains, we see that the heights of the mountains significantly affect the propagation of the pollutants and their negative effects on the observed area over time. The pollutants are all blown out of the domain for the flat domain very quickly compared to the other cases. The observed area experiences a high concentration of pollutants shortly after they are emitted. However, it quickly plummets and equals 0 within $80 \mathrm{~s}$. We see that the pollutants stay in the domain longer with the existence of the mountains. The mountain on the left obstructs the wind blowing from the left-hand side of the domain. Due to the pressure, the wind will recirculate in the area between the mountains. As a result, the residue of the pollutants will reach the observed area slower but remain in the domain for a longer time. This results in long-term effects on individuals living in the observed area. The mountain on the right-hand side bounces the pollutants back to the middle of the domain. The higher the mountain is, the less the pollutants can flow over it. If both mountains are equally tall, the area is affected by the pollutants for a longer time. For the case where one mountain is higher than the other, the pollutants are blown out of the observed area quickly if they move into the observed area from the shorter mountain.

Despite the long-lasting residue, being situated in the middle of the mountains is not always bad if the concentration of the pollutant residue remaining in the domain is not high enough to cause harmful effects. Having a mountain on the windward side can slow down when the pollutants reach the observed area and reduce the highest concentration it suffers.

These computations are just illustrations of the techniques for real-world problems. The model can be easily extended for other problems such as those with multiple mountains, tall buildings, or pollutant generators. One can change how the pollutants are emitted by modifying the boundary condition of the generator. The model can also be applied to investigate how the pollutions are generated outside of the valley to see how the mountains will obstruct them. This work would help factory owners, urban planners, and regulators come up with proper plans to reduce the harmful effects of pollution on people.

\section{Acknowledgements}

The authors would like to thank the Development and Promotion of Science and Technology Talents Project (DPST) scholarship for the financial support. Furthermore, the $2^{\text {nd }}$ and $3^{\text {rd }}$ authors would like to thank Prof. Dr. Peter Bastian for the valuable discussions during their visits to the scientific computing group of the Interdisciplinary Center for Scientific Computing (IWR), Heidelberg University, in winter 2018 and summer 2019. We would also like to thank the editor and the reviewers for their thoughtful comments and efforts towards improving our manuscript. Any remaining errors are the sole responsibility of the authors.

\section{References}

[1] M Khafaie, C Yajnik, S Salvi and A Ojha. Critical review of air pollution health effects with special concern on respiratory health. J. Air Pullut. Health 2016; 1, 123-36.

[2] World Health Organization, available at: https://www.who.int, accessed March 2020.

[3] T Roberts, M Unsworth and D Ormrod. Effects of gaseous air pollution in agriculture and horticulture. J. Appl. Ecol. 1983; 20, 704.

[4] JNB Bell and M Treshow. Air pollution and plant life. Wiley, West Sussex, 2003. 
[5] J Burney and V Ramanathan. Recent climate and air pollution impacts on Indian agriculture. In: Proceedings of the National Academy of Sciences, Washington DC, 2014, p. 16319-24.

[6] M Ghani, K Gueraoui and S Men-La-Yakhaf. Mathematical modeling and numerical of the atmospheric pollution at the level of free surface. In: Proceedings of the $13^{\text {th }}$ Congrès de Mécanique. Morocco, 2017.

[7] R Temam. The Navier-Stokes equations: theory and numerical analysis. American Mathematical Society Chelsea Publishing, Rhode Island, 2001.

[8] V Girault and PA Raviart. Finite element approximation of the Navier-Stokes equations. SpringerVerlag, Berlin, 2008.

[9] N Pochai. A finite element solution of the mathematical model for smoke dispersion from two sources. Int. J. Math. Comput. Sci. 2011; 5, 1110-4.

[10] KJ Bathe. Finite element procedures. Klaus-Jürgen Bathe, USA, 2014.

[11] JN Reddy. Introduction to the finite element method. McGraw-Hill Education, New York, 2019.

[12] OC Zienkiewicz, RL Taylor and DD Fox. The finite element method. Elsevier ButterworthHeinemann, Amsterdam, 2014.

[13] Y Hasbani, E Livne and M Bercovier. Finite elements and characteristics applied to advectiondiffusion equations. Comput. Fluid. 1983; 11, 71-83.

[14] P Musik and K Jaroensutasine. Two dimensional lattice boltzmann method for cavity flow simulation. Walailak J. Sci. Tech. 2004; 1, 53-70.

[15] M Blatt, A Burchardt, A Dedner, C Engwer, J Fahlke, B Flemisch, C Gersbacher, C Gräser, F Gruber, C Grüninger, D Kempf, R Klöfkorn, T Malkmus, S Müthing, M Nolte, M Piatkowski and O Sander. The distributed and unified numerics environment, version 2.4. Arch. Numer. Software 2016; 4, 13-29.

[16] P Bastian, M Droske, C Engwer, R Klöfkorn, T Neubauer, M Ohlberger and M Rumpf. Towards a unified framework for scientific computing. In: Proceedings of the $15^{\text {th }}$ International Conference on Domain Decomposition Methods. Berlin, Germany, 2005.

[17] C Geuzaine and JF Remacle. Gmsh: A 3-D finite element mesh generator with built-in pre- and post-processing facilities. Int. J. Numer. Meth. Eng. 2009; 79, 1309-31.

[18] P Kundu and I Cohen. Fluid mechanics. Elsevier Science, Amsterdam, 2001.

[19] JR Cannon. The one-dimensional heat equation. Cambridge University Press, Cambridge, 2008.

[20] P Bastian, C Engwer, D Göddeke, O Iliev, O Ippisch, M Ohlberger and S Turek. EXA-DUNE: Flexible PDE solvers, numerical methods and applications. In: Proceedings of the European Conference on Parallel Processing. Porto, Portugal, 2014.

[21] F Pasquill. Atmospheric diffusion. Horwood, Chicsester, 1974.

[22] NN Yanenko. The method of fractional steps. Springer-Verlag, Berlin, 1971.

[23] SA Konglok and S Tangmanee. Numerical solution of advection diffusion of an air pollutant by the fractional step method. In: Proceedings of the $3^{\text {rd }}$ National Symposium on Graduate Research, 2002.

[24] S Ulfah, SA Awalludin and Wahidin. Advection-diffusion model for the simulation of air pollution distribution from a point source emission. J. Phys. Conf. Ser. 2018; 948, 12067-75.

[25] P Oyjinda and N Pochai. Numerical simulation of an air pollution model on industrial areas by considering the influence of multiple point sources. Int. J. Diff. Equat. 2019; 2019, 2319831.

[26] S Chomcheon, N Khajohnsaksumeth, B Wiwatanapataphee and X Ge. Modeling and simulation of air pollutant distribution in street canyon area with skytrain stations. Adv. Differ. Equat. 2019; 2019, 459.

[27] Z Zhang, X Xu, L Qiap, D Gong, S Kim, Y Wang and R Mao. Numerical simulations of the effects of regional topography on haze pollution in Beijing. Sci. Rep. 2018; 8, 5504. 